VIII ${ }^{\text {èmes }}$ Journées Nationales Génie Civil - Génie Côtier, Compiègne, 7-9 septembre 2004

\title{
Modélisation de la houle sur une plage par la résolution en volumes finis des équations de Serre
}

\author{
Rodrigo Cienfuegos $^{(\mathrm{a})}$, Eric Barthélemy ${ }^{(\mathrm{b})}$ et Philippe Bonneton ${ }^{(\mathrm{c})}$ \\ ${ }^{(a)}$ Doctorant, ${ }^{(b)}$ Professeur à l'I.N.P. Grenoble, \\ email: rodrigo.cienfuegos@hmg.inpg.fr \\ email: eric.barthelemy@hmg.inpg.fr \\ Laboratoire des écoulements Géophysiques et Industriels \\ BP 53, 38041 Grenoble cedex9, France. \\ ${ }^{(c)}$ Chargé de recherche du CNRS, email: p.bonneton@epoc.u-bordeaux1.fr \\ Département de Géologie et d'Océanographie \\ Université de Bordeaux I \\ Av. des Facultés 33405 Talence, France.
}

\section{Résumé}

Un schéma de résolution numérique en volumes finis pour un système d'équations de Serre étendu est présenté. En utilisant des solutions analytiques tests nous montrons que celuici fournit une erreur de discrétisation globale de $\left[\mathrm{O}\left(\Delta \mathrm{x}^{4}\right), \mathrm{O}\left(\Delta \mathrm{t}^{4}\right)\right]$ et que le traitement des conditions aux limites (trait de côte mobile et absorption-génération d'ondes au large) doit permettre d'aborder l'étude de l'évolution morpho dynamique d'un profil de plage.

La comparaison des résultats numériques avec des mesures expérimentales de propagation d'ondes solitaires sur une plage confirme la capacité des équations de Serre à reproduire les non linéarités associées au raidissement des ondes dans la zone de levée et l'amplitude limite de celles-ci.

\begin{abstract}
$\underline{\text { Abstract }}$
A numerical model for solving an extended system of Serre equations using the finite volume method is presented. Comparisons with some analytical solutions show that the numerical model reaches a global discretization error of $\left[\mathrm{O}\left(\Delta \mathrm{x}^{4}\right), \mathrm{O}\left(\Delta \mathrm{t}^{4}\right)\right]$ and that the numerical implementation of boundary conditions (moving shoreline and absorbinggenerating boundary at the entrace) should be sufficient to move on into cross-shore beach morphology studies. Furthermore, comparisons between numerical and experimental solitary wave shoaling on a beach confirm the capacity of Serre equations to deal with strong non linearities.
\end{abstract}

\section{Introduction}

$\mathrm{Au}$ cours des dernières décennies, d'importants efforts ont abouti à des améliorations significatives des équations de la famille de Boussinesq qui servent à décrire la progagation de la houle sur un fond quelconque. Elles constituent à présent un outil mathématique puissant qui permet d'accéder à un certain nombre d'informations nécessaires (asymétries des houles, vitesses, etc...) pour effectuer une prédiction correcte de l'évolution morphologique des plages. Dans le contexte des applications côtières, il est maintenant possible dans ce type de modèles de bien représenter la levée non linéaire, la dispersion en fréquence et la dissipation d'énergie par déferlement qui a lieu dans la zone de «surf»

Dans la pratique, la résolution numérique de ce type d'équations a été principalement abordée par des schémas aux différences finies centrées con $\backslash$ c $\{c\}$ us pour minimiser la dispersion numérique qui peut éventuellement l'emporter sur la vraie dispersion physique du 
système (voir par exemple Wei et coll. ${ }^{1}$ ). Similairement, quelques exemples de l'application de la méthode des éléments finis à ce type d'équations existent (Antunes do Carmo et coll. ${ }^{2}$ ). Cependant, l'utilisation de la méthode des volumes finis n'a été abordée que récemment (Bradford et Sanders ${ }^{3}$ ).

Nous décrivons dans ce travail une résolution numérique des équations de Serre, avec une correction de la dispersion basée sur l'approche de Madsen et Schäffer ${ }^{4}$, par un schéma de volumes finis à grilles décalées pour la discrétisation spatiale; l'intégration dans le temps s'effectue à l'aide d'une méthode de Runge-Kutta d'ordre 4.

Dans la première partie nous présentons la forme conservative des équations que nous avons choisies de résoudre numériquement, pour aborder ensuite les aspects pratiques de la mise en oeuvre du schéma de résolution. Finalement, nous évaluons les capacités de la nouvelle méthode en comparant les résultats avec quelques solutions analytiques et expériences en laboratoire.

\section{Equations de Serre étendues}

En s'intéressant aux ondes longues à forte courbure, F. Serre ${ }^{5}$ obtient une théorie plus générale que celle décrite par les équations de Boussinesq. Il en déduit alors, sur un fond graduellement varié, un système d'équations moyennées sur la colonne d'eau qui incorpore les effets convectifs associés à une forte accélération verticale du fluide. Plus tard, Seabra Santos et coll. ${ }^{6}$ généralisent les équations de Serre à un fond quelconque. Ces équations constituent en fait un modèle de Boussinesq sans restrictions sur les non linéarités $\left(\varepsilon=\mathrm{a} / \mathrm{h}{ }_{0}\right)$ où tous les termes en $\mathrm{O}\left(\sigma^{2}\right)$ sont retenus (avec $\sigma=\mathrm{kh}_{0}$, a et $1 / \mathrm{k}$ étant des grandeurs représentatives de l'amplitude et de la longueur de l'onde, tandis que $\mathrm{h}_{0}$ est une mesure caratéristique de la profondeur d'eau). Il s'agit donc d'un système d'équations équivalent (mise à part de la stratégie de correction de la dispersion) à celui présenté quelques années plus tard par Wei et coll. . .

Dans le cadre des applications côtières, il faut remarquer que les hypothèses qui conduisent aux équations de Serre imposent des restrictions importantes concernant leur validité dans la limite de l'eau profonde. En particulier, la vitesse de phase des ondes courtes $\left(\mathrm{kh}_{0} \sim \mathrm{O}(1)\right)$ est mal reproduite. En utilisant une approche étudiée par Madsen et Schäffer ${ }^{4}$ qui consiste à rajouter des termes d'ordre $\mathrm{O}\left(\sigma^{4}\right)$ afin de modifier la relation de dispersion linéaire du système, il est possible d'étendre la limite d'application des équations de Serre à l'eau profonde. Il s'agit de rajouter à l'équation de conservation de la quantité de mouvement le terme,

$$
B(x, t)=-\alpha \xi^{2}\left[u_{x x t}+\left(u u_{x}\right)_{x x}+g(h+\xi)_{x x x}\right]
$$

avec $\alpha$ un coefficient à ajuster. Nous avons introduit ici les coordonnées horizontale $\mathrm{x}$ (avec l'axe Ox confondu avec la position de la surface libre au repos), temporelle $t$, l'équation pour le fond $\mathrm{z}=\xi(\mathrm{x})$ (avec $\mathrm{z}$ la coordonnée verticale positive vers le haut), la hauteur totale de la colonne d'eau $h(x, t)=\eta(x, t)-\xi(x)$ ( $\eta$ étant la dénivellation de la surface libre), la vitesse horizontale moyennée sur la colonne d'eau $\mathrm{u}(\mathrm{x}, \mathrm{t})$ et l'accélération de la gravité $\mathrm{g}$.

En incorporant le terme (1) dans les équations présentées par Seabra Santos et coll. ${ }^{6}$, il est possible d'écrire le système d'équations de Serre étendu sous la forme conservative suivante,

$$
\begin{aligned}
& h_{t}^{+(u h)_{x}=0} \\
& q_{t}^{+(u q+F)_{x}=S}
\end{aligned}
$$


$q=(1+r) u-\frac{1}{3 h}\left(h^{3} u_{x}\right)_{x}-\alpha \xi_{2} u_{x x}$

avec,

$F=g(h+\xi)-\frac{1}{2} u^{2}\left(1+\xi_{x}^{2}\right)+u \xi_{x} h u_{x}-\frac{1}{2}\left(h u_{x}\right)^{2}-\alpha \xi^{2}\left[u_{x}^{2}+g(h+\xi)_{x x}\right]$,

$S=-2 \alpha \xi \xi_{x}\left[u u_{x}+g(h+\xi)_{x}\right]_{x}, \quad r=\left(h_{x}+\xi_{x}\right) \xi_{x}+\frac{1}{2} h \xi_{x x}$

Nous remarquons que la correction introduite permet d'approximer la relation de dispersion de Stokes à l'aide d'un développement de Padé [2,2] quand $\alpha=1 / 15$

Il est intéressant de noter que contrairement aux équations de Boussinesq, sur fond horizontal et pour $\alpha=0$, ce système d'équations admet une solution explicite sous forme d'une onde solitaire qui correspond à la solution de Rayleigh. Nous nous servirons plus tard de cette propriété pour étudier l'ordre réel de convergence de notre schéma numérique. En effet, dans le cas général, la résolution du système (2)-(4) doit faire appel au calcul numérique. Or, cette forme conservative des équations de Serre est particulièrement adaptée à une discrétisation en volumes finis et sera exploitée dans la section suivante.

\section{Résolution numérique}

La méthode des volumes finis consiste à diviser l'espace de définition du problème à l'aide de volumes élémentaires et d'intégrer les équations sur chacun d'eux. Les noeuds où sont calculées les variables correspondent aux centroïdes de chaque volume et une interpolation est mise en place pour estimer leurs valeurs sur les faces. Les intégrales de surface et de volume doivent être estimées numériquement à l'aide de formules de quadrature.

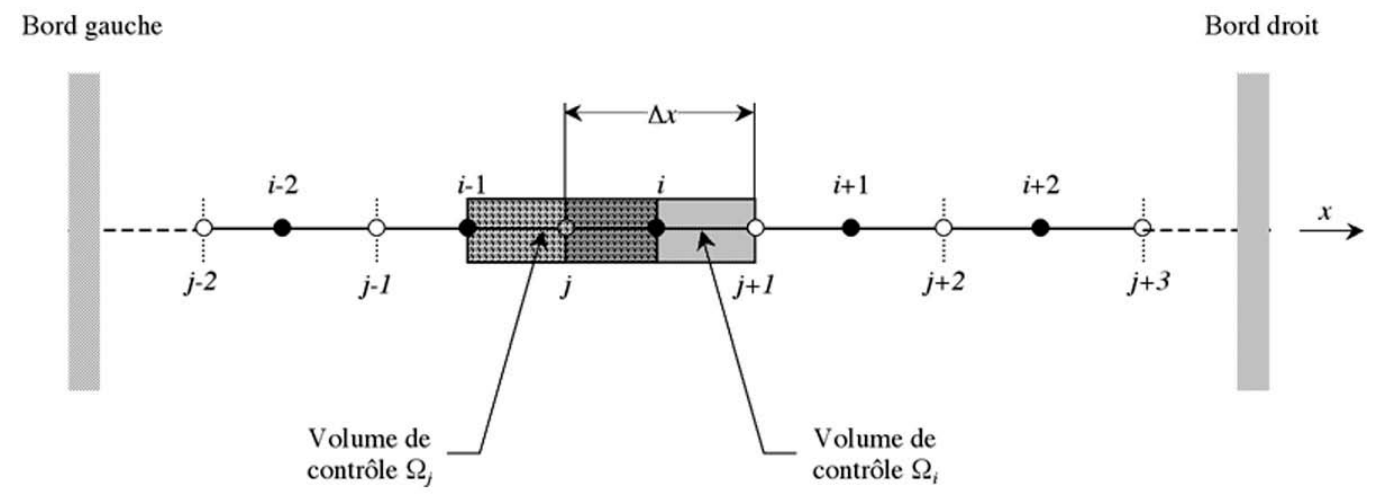

Figure 1 Définition schématique de la grille décalée de discrétisation : $h$ et $q$ sont définis sur les noeuds $i$, u est définie sur les noeuds $j$

\subsection{Discrétisation spatiale: schéma à grilles décalées}

Afin de diminuer le nombre d'interpolations requises et d'améliorer en même temps la résolution numérique des composantes de courte longueur d'onde (Durran ${ }^{7}$ ), nous allons intégrer les équations (2) et (3) sur le volume de contrôle $\Omega i$, tandis que l'équation (4) le sera sur le volume $\Omega \mathrm{j}$ (Figure. 1). L'intégration sur les volumes de contrôle respectifs permet d'écrire,

$\frac{d}{d t}\left(\int_{x j}^{x j+1} h d x\right)+\left.(u h)\right|_{x j} ^{x j+1}=0$ 


$$
\begin{aligned}
& \frac{d}{d t}\left(\int_{x j}^{x j+1} q d x\right)+\left.(u q)\right|_{x j} ^{x j+1}+\left.F\right|_{x j} ^{x j+1}=\int_{x j}^{x j+1} S d x \\
& \int_{x i-1}^{x i} q d x=\int_{x i-1}^{x i} \mid(1+r) u-\frac{1}{3 h}\left(h_{3} u_{x}\right)_{x}-\alpha \xi_{2} u_{x x} d x
\end{aligned}
$$

avec $\int_{x a}^{x b}=\int(x b)-\int(x a)$. Afin de garantir une erreur de discrétisation $\mathrm{O}\left(\Delta \mathrm{x}^{4}\right)$ nous utilisons la méthode de Simpson pour évaluer les intégrales et une interpolation cubique pour estimer les valeurs des variables sur les frontières de chaque volume de contrôle.

L'intégration des termes non-conservatifs de la fonction $S$ dans l'équation (6) et du membre de

droite de l'équation (7) doit être approximée. En prenant comme exemple le 2ème terme de ce dernier, nous écrivons,

$-\int_{x i-1}^{x i} \frac{1}{3 h}\left(h_{3} u_{x}\right)_{x} d x \approx-\left.\frac{1}{3 h_{j}}\left(h_{3} u_{x}\right)\right|_{x i-1} ^{x i}$

Les autres termes sont traités de façon analogue. D'un autre côté, les dérivées par rapport à $\mathrm{x}$ sont estimées à l'aide de l'approximation suivante qui fournit une erreur de discrétisation $\mathrm{O}\left(\Delta \mathrm{x}^{4}\right)$ (Ferziger et Peric $\left.{ }^{8}\right)$,

$u x|i-1+4 u x| i+u_{x} \mid i+1=\frac{3}{\Delta x}\left(u_{i+1}-u_{i-1}\right)$

Le système matriciel global issu de ce processus est formé de matrices pentadiagonales et peut être exprimé de la manière suivante :

$$
\begin{aligned}
& A \frac{d h_{i}}{d t}+M\left(u_{j}\right) h_{i}=0 \\
& A \frac{d q_{i}}{d t}+M\left(u_{j}\right) q_{i}+F\left(h_{i}, u_{j}\right)=S\left(h_{i}, u_{j}\right) \\
& B q_{i}=G\left(h_{i}\right) u_{j}
\end{aligned}
$$

\subsection{Intégration dans le temps et conditions aux limites}

Pour l'intégration dans le temps des équations (9) et (10) nous utilisons une méthode de Runge-Kutta d'ordre 4 qui donne en théorie une erreur $\mathrm{O}\left(\Delta \mathrm{t}^{4}\right)$. A la fin de chaque étape de calcul, le vecteur des vitesses nodales $\boldsymbol{u}_{j}$ est calculé à partir de l'équation (11).

Dans le contexte de la modélisation des évolutions morphodynamiques des plages, nous sommes amenés à définir des conditions aux limites appropriées. En particulier nous devons être capables, au large, de faire entrer une houle mais aussi de permettre aux ondes réfléchies par la plage de sortir du domaine sans provoquer de réflexions parasites. D'un autre côté, si l'on veut étudier la formation de barres de réflexion, il est indispensable de bien reproduire la dynamique dans la zone de «swash», en particulier le «runup», et le «run-down»

La mise en oeuvre numérique de telles conditions aux limites est un sujet complexe qui ne pourra pas être traité ici. Nous signalons simplement que la condition d'absorption-génération d'ondes au large est basée sur une décomposition quasi-hyperbolique du système de Serre qui permet l'utilisation des équations caractéristiques à condition que pour l'onde sortante, $\mathrm{O}\left(\varepsilon \sigma^{2}\right)<<1$. Or, dans le cas de réflexion sur une plage à pente relativement douce, cette condition est souvent vérifiée. 
Le traitement du trait de côte mobile s'effectue en utilisant une technique d'extrapolation similaire à celle présentée par Lynett et coll ${ }^{9}$.

\section{$\underline{\text { 4.Validation }}$}

\subsection{Propagation d'une onde solitaire sur fond horizontal}

En utilisant la solution analytique d'onde solitaire des équations de Serre, nous étudions ici l'ordre de convergence réel du schéma numérique. Pour ce faire nous estimons l'erreur commise lorsqu'une onde solitaire d'amplitude relative $\mathrm{a} / \mathrm{h}_{0}=0.3$ se propage sur deux longueurs caractéristiques $\lambda$ (distance pour laquelle $\eta / h_{0} \geq 0.001$ ), et pour différents pas de discrétisation. L'erreur est estimée à l'aide de la norme,

$$
L_{1}=\frac{1}{N} \sum_{i=1}^{i=N}\left|h_{i}^{a}-h_{i}^{c}\right|
$$

avec $\mathrm{h}^{\mathrm{a}}$ la solution analytique et $\mathrm{h}^{\mathrm{c}}$ la solution estimée numériquement. Les résultats sont présentés sur la figure 2 .
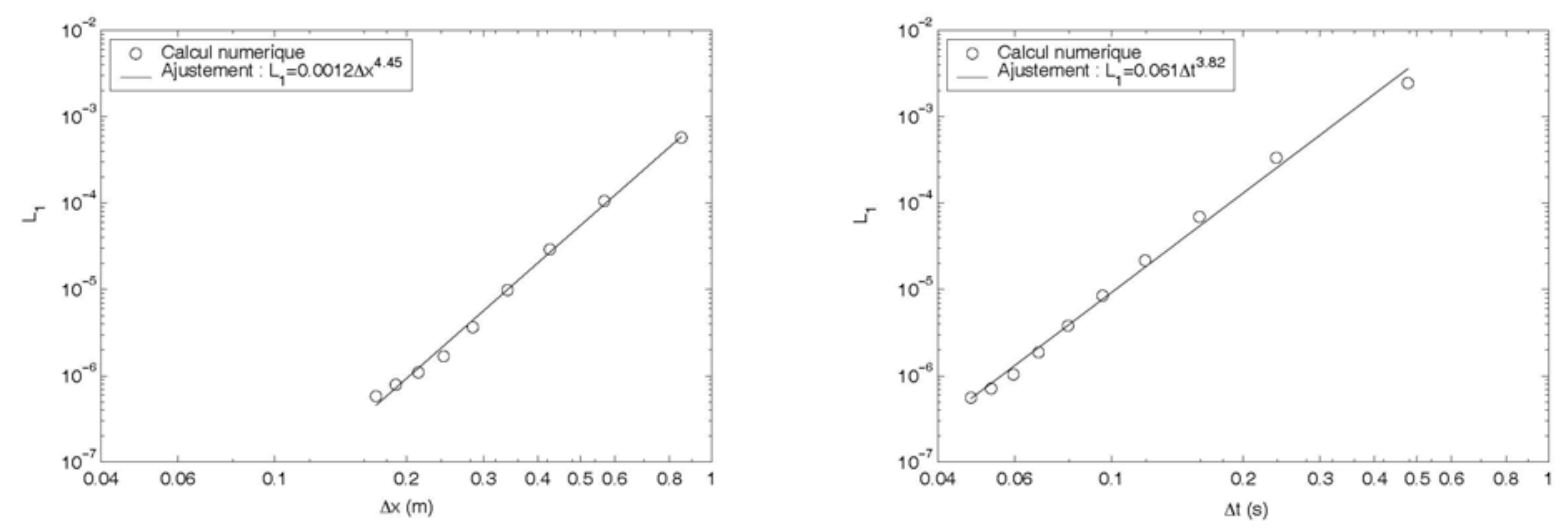

Figure 2: Erreur de discrétisation L1 en fonction de $\Delta x$ et $\Delta t$ pour la propagation d'une onde solitaire à $a / h_{0}=0.3$ sur deux longueurs caractéristiques $\lambda$

Le calcul confirme l'ordre de convergence théorique du schéma en $\mathrm{O}\left(\Delta \mathrm{x}^{4}\right)$ et $\mathrm{O}\left(\Delta \mathrm{t}^{4}\right)$. D'autre part, des tests effectués sur la propagation d'ondes solitaires avec nonlinéarités importantes $\left(\mathrm{a} / \mathrm{h}_{0}=0.6\right)$ sur une dizaine de longueurs caractéristiques montrent que le schéma est très peu dissipatif et conserve bien la forme et la célérité de l'onde même pour une discrétisation de $\lambda / \Delta \mathrm{x}=20$.

\subsection{Validation du traitement des conditions aux limites}

Ici, la solution analytique de Carrier et Greenspan ${ }^{10}$ qui décrit une onde stationnaire non déferlante sur une plage plane sera utilisée pour valider à la fois la condition aux limites de trait de côte mobile et d'absorption-génération d'ondes au large. Pour cela, nous faisons entrer une onde sinusoïdale d'amplitude $\mathrm{a}=0.003 \mathrm{~m}$ et de période $\mathrm{T}=10 \mathrm{~s}$ sur une plage de pente $1 / 25$ à partir d'une section située à $30 \mathrm{~m}$ de la ligne de côte (la profondeur d'eau dans la partie à fond horizontal est $\mathrm{h}_{0}=0.5 \mathrm{~m}$ ). Le calcul se poursuit alors jusqu'à atteindre la solution stationnaire qui est la conséquence de la superposition d'ondes incidentes et réfléchies. Les termes dispersifs ne sont pas pris en compte dans le calcul afin de pouvoir comparer les résultats numériques avec la solution analytique. La figure 3 montre une concordance remarquable entre la théorie et le calcul numérique. 


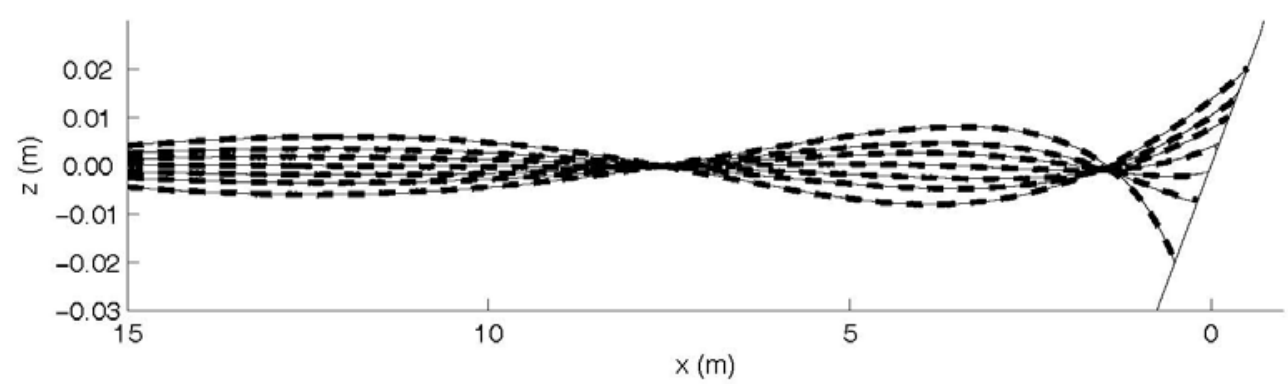

Figure3: Déplacements de la surface libre de l'onde stationnaire. Théorie de Carrier et Greenspan ${ }^{10}$ (-) et résolution numérique des éqs. (2)-(4) (--) avec $\Delta x=0.04$ et $\Delta t=0.02 \mathrm{~s}$

\subsection{Levée non linéaire d'une onde solitaire}

Le dernier test que nous effectuons consiste à propager une onde solitaire sur une plage de pente uniforme $1 / 30$ et de profondeur $\mathrm{h}_{0}=25 \mathrm{~cm}$ dans la partie à fond horizontal. Nous confrontons le calcul numérique à quelques expériences réalisées dans le canal à houle du LEGI $(36 \mathrm{~m})$. Les mesures des déplacements de la surface libre s'effectuent à l'aide de six sondes résistives réparties dans le canal (la lère située sur la partie horizontale et les 5 autres dans la zone de levée). Il s'agit ici d'un test important concernant la performance du modèle en présence de fortes non linéarités. On note dans la figure 4 un excellent accord.
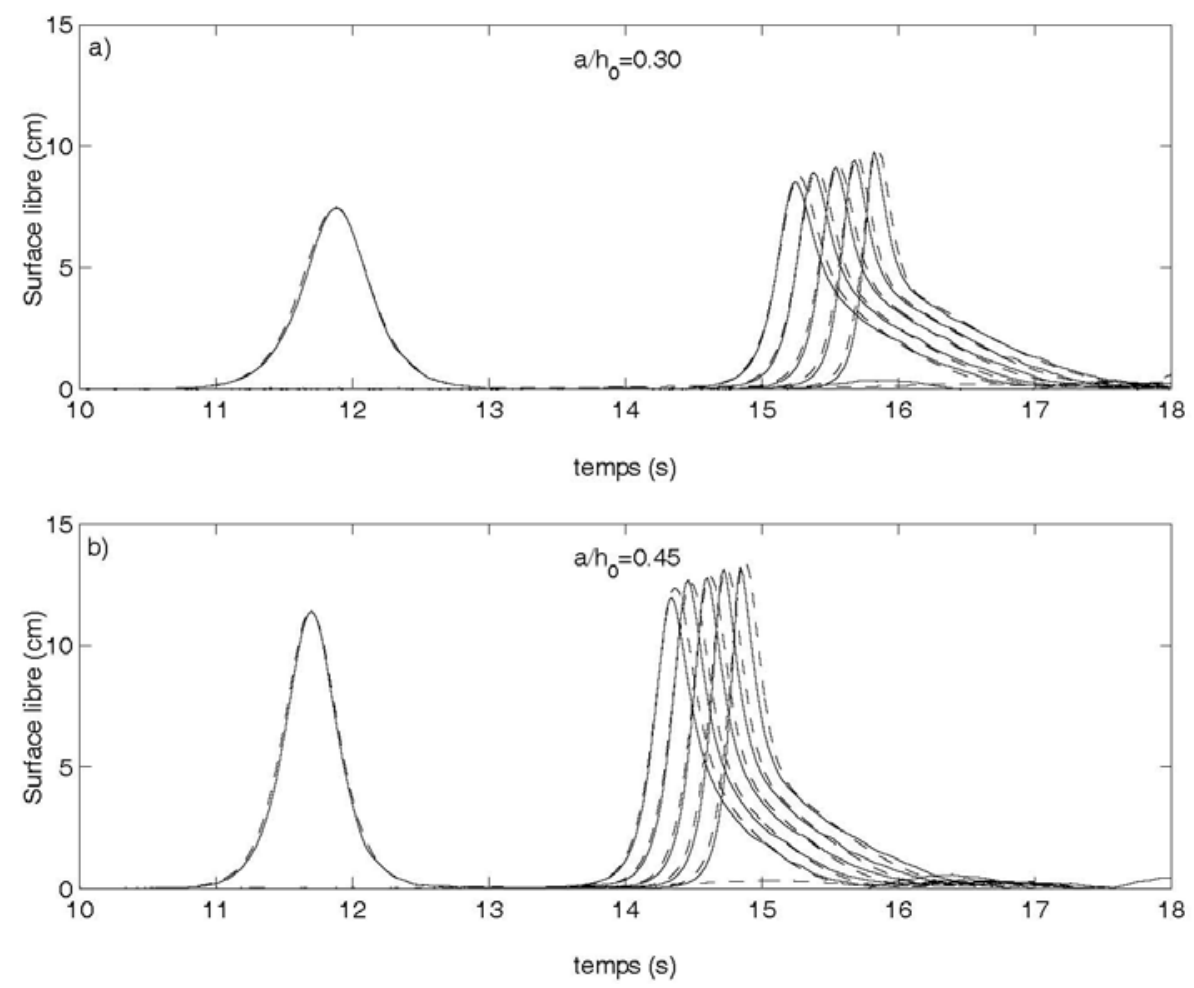

Figure 4: Déplacement de la surface libre mesuré (-) et calculé numériquement (--) dans différentes sections du canal. a/h $h_{0}$ correspond à l'amplitude relative de l'onde incidente (mesurée par la lère sonde située à une distance $x=9.94 \mathrm{~m}$ du trait de côte).

Localisation des autres sondes :

a) $x=3.980,3.765,3.510,3.290,3.052 \mathrm{~m}$;

b) $x=4.910,4.695,4.444,4.220,3.982 \mathrm{~m}$ 


\section{Conclusions}

Une nouvelle méthode de résolution numérique qui utilise une discrétisation en volumes finis a été appliquée avec succés à une version étendue des équations de Serre. Ces équations incorporent un terme de correction des propriétés dispersives qui permet de reproduire correctement la vitesse de phase d'ondes se propageant même en eau profonde $\left(\mathrm{kh}_{0}=3 \sim 4\right)$. Des essais préliminaires montrent que la méthode numérique est robuste, très peu dissipative et engendre une erreur de discrétisation globale de $\left[\mathrm{O}\left(\Delta \mathrm{x}^{4}\right), \mathrm{O}\left(\Delta \mathrm{t}^{4}\right)\right]$. De plus, l'utilisation de conditions aux limites adaptées aux études de l'évolution morphologique des plages a été validé.

D'un autre côté, une comparaison de résultats expérimentaux et numériques dans le cas de la propagation d'ondes solitaires sur une plage confirme la bonne performance des équations de Serre dans un contexte de forte non linéarité. Cette propriété devrait permettre d'obtenir une estimation correcte des amplitudes dans la zone de levée et sera fondamentale pour bien localiser le point de déferlement. Afin d'introduire la dissipation turbulente qui se produit dans la zone de «surf», et de disposer ainsi d'un modèle hydrodynamique applicable depuis le large et jusqu'au trait de côte, nous évaluons actuellement les alternatives existantes (rouleau de déferlement, viscosité turbulente).

\section{Références}

1.G.Wei, J.T.Kirby, and R.Subramanya. Afully non linear Boussinesq model for surface waves. Part 1. Highly unsteady waves. J.Mech., 29: 71-92, 1995.

2.J.S.Antunes do Carmo, F.J.Seabra Santos, and E.Barthelemy. Surface waves propagation in shallow wate: A finite element model. Int.J.Numer.Meth. in Fluids, 16: 447459, 1993.

3.S.F.Bradford and B.F.Sanders. Finite-volume models for unidirectional, non linear, dispersive waves; J.Waterway, Port, Coast and Oc. Eng, 128(4):173-182, 2002.

4.P.A.Madsen and H.A.Schäffer. Higher-order Boussinesq-type equations for surface gravity waves: derivation and analysis. Phil. Trans. Roy. Soc. London, A(356): 3123-3184, 1998.

5.F.Serre. Contribution à l'étude des écoulements permanents et variables dans les canaux. Houille Blanche, 8 :374-388, 1953.

6.F.J.Seabra Santos, D.P.Renouard, and A.M.Temperville. Numerical and experimental study of the transformation of a solitary wave over a shelf or isolated obstacle. J.Fluid Mech, 176:117-134, 1987.

7.D.R.Durran. Numerical Methods for wave Equations in Geophysical Fluid Dynamics. Springer, $1^{\text {st }}$ edition, 1999.

8.J.H.Ferziger and M.Peric. Computational Methods for Fluid Dynamics. Springer, $3^{\text {rd }}$ edition, 2002.

9.P.J.Lynett, T.-R. Wu, and P.L.-F. Liu. Modeling wave runup with depth-integrated equations. Coastal Eng., 46: 89-107, 2002.

10.G.F.Carrier and H.P.Greenspan. Water waves of finite amplitude on a sloping beach. J.Fluid Mech, 4: 97-109, 1958. 